\title{
Leveraging the electronic health record to implement genomic medicine
}

\author{
Iftikhar J. Kullo, MD¹, Gail P. Jarvik, MD, PhD ${ }^{2,3}$, Teri A. Manolio, MD, PhD, \\ Marc S. Williams, MD ${ }^{5}$ and Dan M. Roden, MD $^{6,7}$
}

The use of whole-genome/exome sequence in select clinical settings to improve health and prevent disease, considered to be improbable a few years ago, is now approaching reality. However, the advent of "next-generation" sequencing technologies presents a paradox: the prospect of managing everincreasing amounts of genomic data is in itself an obstacle to the vision of genome-informed health care. Here, we present our view that the solution to integrating these data into the flow of health care lies in a genome-enabled electronic health record (EHR). ${ }^{1}$ Such EHRs would receive, store, and present complex genomic information for clinical use, incorporate clinical decision support to help providers practice individualized medicine, and provide links to relevant knowledge resources. Although adoption of EHRs is increasing, the majority of EHR systems are not configured to deal with genetic data, ${ }^{2}$ particularly data from whole-genome/exome sequences. This article summarizes the challenges in implementing genomic medicine using the EHR and discusses the role various stakeholders might play in addressing these challenges.

\section{HEALTH-CARE PROVIDERS AND PATIENTS}

Health-care providers will need to learn more about the "new" genetics and recognize that genetic variants may influence response to drugs and susceptibility to common "complex" as well as Mendelian diseases. An optimized EHR will leverage genomic knowledge and linked resources to facilitate interpretation and application of genomic data in the clinical setting. Interpretation of genomic data will be influenced by family history, and thus improved methods of acquiring and representing family history in the EHR are needed. Successful integration of genomics into clinical care will require minimizing the burden on providers by using tools such as pictograms and decision aids to communicate genomic information to patients. Web-based, secure, and user-friendly portals can empower patients to know more about how their genomic information is used for assessing disease risk, prognostication, and tailored drug therapy. Providers and patients should be aware that the genetic risk for diseases is probabilistic, not deterministic, and often can be modified by lifestyle and environmental factors.
Communication of genome sequencing results should take into consideration patients' preferences about what risk information they wish to know and have placed in their EHR.

\section{BIOETHICISTS, GENETIC COUNSELORS, AND POLICY MAKERS}

There is an emerging consensus about patients' right to receive or decline to receive their own data, and patients should be empowered to choose to seek and use such data in an informed manner. However, the right to not receive or to decline particular types of information may clash with the obligations of the physician when critically important health information is available. Before genome sequencing, patients will require counseling about the potential burden of genetic knowledge and implications for family members or reproductive choices. Perceived concerns about insurance and employability will remain despite the passage of the Genetic Information Nondiscrimination Act, especially in the realm of long-term care and life and disability insurance. Once genome sequencing is completed, patients need to be educated about the interpretation and implications, and the options available for following up the results. Such pre- and post-test counseling could be conducted by medical geneticists or genetic counselors using Web-based patient portals as aids; however, due to shortage of such providers, training of other care providers may be necessary. As for all health data, it is important to maintain confidentiality of genetic data and regulate third-party access. Patients are likely to be concerned about how genetic testing would be paid for and how such testing may affect insurability. Finally, reporting of genome sequencing results might be constrained by intellectual property restrictions, and this issue will need to be addressed by regulatory bodies.

\section{CLINICAL MOLECULAR GENETICISTS}

Variants to be reported from clinical genome sequencing must be assayed in a Clinical Laboratory Improvement Amendments-certified laboratory. Clinically actionable variants from the sequence would then be placed in the EHR as structured data with accompanying interpretation. Whether

${ }^{1}$ Division of Cardiovascular Diseases, Mayo Clinic, Rochester, Minnesota, USA; ${ }^{2}$ Department of Medicine (Medical Genetics), University of Washington, Seattle, Washington, USA; ${ }^{3}$ Department of Genome Sciences, University of Washington, Seattle, Washington, USA; ${ }^{4}$ National Human Genome Research Institute, Bethesda, Maryland, USA; ${ }^{5}$ Geisinger Medical Center, Danville, Pennsylvania, USA; ${ }^{6}$ Department of Medicine, Vanderbilt University School of Medicine, Nashville, Tennessee, USA; ${ }^{7}$ Department of Pharmacology, Vanderbilt University School of Medicine, Nashville, Tennessee, USA. Correspondence: Iftikhar J. Kullo (kullo.iftikhar@mayo.edu) 
"actionable" variants identified by next-generation sequencing would need validation on an orthogonal platform, before reporting, is unclear. It is possible that advances in next-generation sequencing platforms will improve analytic validity to the point of obviating need for confirmatory testing. Health Level Seven is a standard for transferring data between information systems, and the Health Level Seven organization has published an implementation guide outlining health-care data standards for reporting structured genetic test results. ${ }^{3}$ Regulatory guidance for genome sequencing tests may facilitate its use in the clinical setting. An early task would be to develop guidelines for how and under what circumstances whole-genome/exome sequencing and potential future re-analyses would be reimbursed.

\section{BIOINFORMATICIANS}

Individual genomes contain large numbers of variants that are not in reference genomes, and their role in disease susceptibility or drug response is unknown. Extensive effort is needed to annotate variants of uncertain clinical significance present in whole-genome data sets and to identify "actionable variants" to report to providers. This could be greatly facilitated by a curated database of actionable variants that stores validated genotypephenotype associations, such as proposed in a recent request for applications (HG-12-016) issued by the National Human Genome Research Institute. As our knowledge base increases, genome sequence data may need to be reinterpreted periodically, creating the need to recontact patients and their providers. If new genomic information does not meet a minimal threshold for clinical utility and validity, there is little point in reporting it (and, indeed, potential harm in doing so).

\section{INFORMATION TECHNOLOGY SPECIALISTS}

Eventually, germline whole-genome sequencing has the potential to be an efficient one-time test, provided the sequence data are highly accurate and can be stored and accessed as clinically indicated at various times in an individual's life span. However, placing whole-genome sequence data sets in individual EHRs may prove problematic. One solution is to store sequence data outside the EHR and place a "minimal" data set of variants of established clinical utility in the EHR for linkage to clinical decision support. The sequence could be re-interrogated as new genotype-phenotype associations are identified and additional variants of clinical relevance can then be ported to the EHR to be available for clinical decision making. Alternatively, such interrogation can be need-based, i.e., ordered by a care provider to help answer a specific clinical question.

\section{MEDICAL INFORMATICS EXPERTS}

To enable providers to deal with complex and rapidly evolving genomic data, the EHR will need to be a source of guidance at the point of care. By linking curated, annotated, and actionable genetic variants to clinical decision support, specific recommendations for further screening/testing and individualized drug therapy could be made. To trigger clinical decision support, genetic variants must be represented in a structured, "machine readable" format. Genomic decision support systems should enable shared decision making by patients and their caregivers and be readily updatable as new, clinically relevant information is acquired. Partnerships between academia and EHR vendors may expedite the development of decision support modules for implementing genomic medicine.

\section{RESEARCHERS}

Whole-genome sequence data, when linked to the rich phenotypic information in the EHR, can identify novel genotype-phenotype associations and advance our knowledge of disease pathophysiology. ${ }^{4}$ Investigators in the Electronic Medical Records and Genomics (eMERGE) Network have demonstrated that phenotypes of interest can be mined for genomic association studies using electronic phenotyping algorithms. ${ }^{5}$ Many research questions could be addressed faster and at lower cost as compared with assembling and phenotyping large cohorts de novo. Patients need to be informed and active partners in advancing EHR-based genomic research, and providers need to ensure that patient privacy and quality of care is maintained. Although many questions might be answered from existing data in EHRs, prospective studies will likely still be needed to inform clinical decision making. Considerable resources are required for such studies and the generalizability of tightly controlled clinical trials is also limited. The research community needs to reach a consensus regarding when EHR-derived data are "good enough".

Individualized medicine based on advances in genomics has the potential to improve the health of both individuals and populations, by enabling early detection of disease, targeted screening, and tailored therapy. A genome-enabled EHR is essential to translate advances in genomics if such improvements are to be realized. Such an EHR would include point-of-care clinical decision support tools, links to genomic knowledge resources, and the ability to query the genome sequence as needed. Active involvement of various stakeholders is needed to enable integration of genomic information into the EHR.

\section{ACKNOWLEDGMENTS}

We thank Erwin Bottinger, Murray Brilliant, Rex Chisholm, Christopher G. Chute, Eric Larson, David H. Ledbetter, Rongling Li, Cathy A. McCarty, and Maureen Smith for helpful suggestions.

\section{DISCLOSURE}

The authors declare no conflict of interest.

\section{REFERENCES}

1. Hoffman MA, Williams MS. Electronic medical records and personalized medicine. Hum Genet 2011;130:33-39.

2. Scheuner MT, de Vries H, Kim B, Meili RC, Olmstead SH, Teleki S. Are electronic health records ready for genomic medicine? Genet Med 2009;11:510-517.

3. HL7 Version 2 Implementation Guide: Clinical Genomics; Fully LOINC-Qualified Genetic Variation Model (US Realm). http://www.hl7.org/implement/standards/ product_brief.cfm?product_id=23.

4. Ritchie MD, Denny JC, Crawford DC, et al. Robust replication of genotypephenotype associations across multiple diseases in an electronic medical record. Am J Hum Genet 2010;86:560-572.

5. Kullo IJ, Fan J, Pathak J, Savova GK, Ali Z, Chute CG. Leveraging informatics for genetic studies: use of the electronic medical record to enable a genomewide association study of peripheral arterial disease. J Am Med Inform Assoc 2010;17:568-574. 